\title{
Early postoperative decrease of albumin is an independent predictor of major complications after oncological esophagectomy: A multicenter study
}

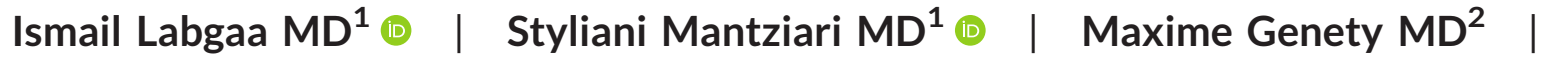 \\ Jessie A. Elliott MD ${ }^{3} \quad$ Satoshi Kamiya MD ${ }^{4}$ | Marianne C. Kalff MD \\ Michaël Winiker $M^{1}$ | Jérôme Pasquier PhD $^{6}$ | Pierre Allemann $M^{1}$ | \\ Marguerite Messier MD ${ }^{2}$ | Mark I. van Berge Henegouwen MD $^{5}$ | \\ Magnus Nilsson MD ${ }^{4}$ ～John V. Reynolds MD $^{3} \quad$ ～Guillaume Piessen MD ${ }^{2}$

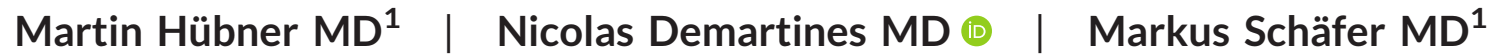

${ }^{1}$ Department of Visceral Surgery, Lausanne University Hospital (CHUV), University of Lausanne (UNIL), Switzerland

${ }^{2}$ Department of Digestive and Oncological Surgery, University of Lille, Claude Huriez University Hospital, Lille, France

${ }^{3}$ Department of Surgery, Trinity Translational Medicine Institute, St. James's Hospital, Dublin, Ireland

${ }^{4}$ Department of Clinical Science, Intervention and Technology (CLINTEC), Division of Surgery, Karolinska Institutet and Karolinska University Hospital, Stockholm, Sweden

${ }^{5}$ Department of Surgery, Amsterdam University Medical Centers, Location Academic Medical Center, University of Amsterdam, Cancer Center Amsterdam, Amsterdam, The Netherlands

${ }^{6}$ Center for Primary Care and Public Health, University of Lausanne, Lausanne, Switzerland

\section{Correspondence}

Markus Schäfer, MD, Department of Visceral Surgery, Lausanne University Hospital CHUV, Rue du Bugnon 46, BH10, CH-1011 Lausanne, Switzerland.

Email: Markus.Schafer@chuv.ch

\begin{abstract}
Background and Objectives: Serum albumin perioperative decrease $(\Delta \mathrm{Alb})$ may reflect the magnitude of the physiological stress induced by surgery. Studies highlighted its value to predict adverse postoperative outcomes, but data in esophageal surgery are scant. This study aimed to investigate the role of $\Delta$ Alb to predict major complications after esophagectomy for cancer.

Methods: Multicenter retrospective study conducted in five high-volume centers, including consecutive patients undergoing an esophagectomy for cancer between 2006 and 2017. Patients were randomly assigned to a training $(n=696)$ and a validation $(n=350)$ cohort. Albumin decrease was calculated on postoperative day 1 and defined as $\Delta \mathrm{Alb}$. The primary endpoint was major complications according to Clavien classification.
\end{abstract}

Results: In the training cohort, esophagectomy induced a rapid drop of albumin. Cut-off of $\Delta$ Alb was established at $11 \mathrm{~g} / \mathrm{L}$ and allowed to distinguish patients with adverse outcomes. On multivariable analysis, $\Delta \mathrm{Alb}$ was identified as an independent predictor of major complications (OR, 1.06; 95\% Cl, 1.01-1.11; $p=.014)$. Higher $\mathrm{BMI}$ and laparoscopy were associated with lower $\Delta \mathrm{Alb}$. Analysis of the validation cohort provided consistent findings.

Conclusions: $\Delta \mathrm{Alb}$ appeared as a promising biomarker after oncological esophagectomy, allowing prediction of potential adverse outcomes.

KEYWORDS

cancer, biomarker, esophagus, surgical oncology 


\section{1 | INTRODUCTION}

Esophageal cancer is the sixth most frequent cause of cancer-related mortality worldwide, with an increased incidence of adenocarcinoma in the Western world related to obesity and uncontrolled reflux disease. ${ }^{1,2}$ Surgery constitutes the cornerstone of curative treatment for non-metastatic lesions, combined with chemo/radiotherapy for locally advanced stages. ${ }^{3,4}$ Although minimally invasive esophagectomy (MIE) may improve short-term outcomes, ${ }^{5,6}$ esophagectomy remains associated with substantial rates of mortality and severe morbidity, which may compromise long-term survival. ${ }^{7,8}$ Early identification of postoperative complications is of utmost importance, as their systemic impact and subsequent risk of mortality may be reduced if they are promptly detected and treated, especially in elderly or frail patients. ${ }^{9}$ In this context, the identification of accurate biomarkers able to predict adverse outcomes early in the postoperative course is of high clinical relevance, as it may help to better anticipate patients' care and to improve their outcomes. Ideal candidate biomarkers need to fulfill the following criteria: performant, early indicative, easy to measure, and cost-effective. ${ }^{10,11}$

Previous studies have suggested postoperative albumin drop $(\triangle \mathrm{Alb})$ as a marker of surgical stress, associated with the risk of postoperative complications after major abdominal surgery. ${ }^{12-16}$ It is already predictive within the first 6-24 $\mathrm{h}$ after surgery and was more robust than other inflammatory biomarkers currently used in daily practice, such as C-reactive protein, which reaches a postoperative peak between postoperative day (POD) 2 and 3.17,18 Albumin as an early predictor of postoperative complications after oncological esophagectomy was reported by Ryan et al. in $2007^{14}$; however, validation by a large-scale study was necessary to recommend its use in clinical practice.

This study aimed to investigate the value of $\Delta \mathrm{Alb}$ to predict major complications after oncological esophagectomy.

\section{2 | MATERIALS AND METHODS}

\subsection{Design and cohorts}

A multicenter retrospective study conducted in five high-volume University Hospitals was conducted: CHUV in Lausanne (Switzerland), CHU of Lille (France), St-James Hospital in Dublin (Ireland), Karolinska University Hospital of Stockholm (Sweden), and Amsterdam University Medical Centers (location AMC) of Amsterdam (Netherlands). Consecutive patients undergoing oncological esophageal resection between 2006 and 2017 were included. Each local Institutional Review Board (IRB) approved the study protocol.

\subsection{Selection of patients}

Inclusion criteria were patients $>18$ years undergoing esophagectomy during the study period. Non-oncological patients and patients with missing preoperative or postoperative values of albumin were excluded. Data were retrieved from institutional databases and transmitted anonymously to the principal investigator for analyses. Collected data included demographics, surgical details, perioperative variables as well as short- and long-term outcomes.

\section{3 | Biomarkers}

Serum albumin concentration was measured in $\mathrm{g} / \mathrm{L}$, in preoperative blood samples taken $<4$ weeks before surgery, as well as postoperatively on POD 1-3. Its fluctuation was defined as $\triangle \mathrm{Alb}$ and calculated on POD 1, as follows: (preoperative value of albumin - albumin value on POD 1). Timepoints were determined based on available prospective data, ${ }^{15}$ as well as to tailor a biomarker being indicative as early as possible. The absolute albumin value on POD 1 (g/L) was separately assessed as a categorical variable, with cutoffs determined at $<20,20-25,25-30$, and $>30 \mathrm{~g} / \mathrm{L}$, based on previous data, suggesting a good correlation with adverse postoperative outcomes. ${ }^{14}$ Subgroup analysis was performed for a subset of patients with available values of white blood cell count (WBC) [G/L] and C-Reactive protein (CRP) (mg/L) on POD 3.

\subsection{Endpoints}

The Clavien classification was used to grade postoperative complications. Minor complications, major complications, and mortality were defined as Grades I-II, III-IV, and V, respectively. ${ }^{19}$ Primary endpoint was major complications whereas secondary endpoints were overall complications, Comprehensive Complication Index $(\mathrm{CCl}),{ }^{20}$ and length of stay (LoS) calculated from the day of surgery.

\section{5 | Statistical analyses}

To obtain training and validation cohorts, patients were randomized in training and validation cohorts, according to a 2:1 ratio. ${ }^{21}$ To do so, we used the randomization system of statistical software. Training cohort was utilized to investigate the perioperative profile of albumin, to identify an optimal cut-off and to explore its predictive value. These findings were thereafter tested in the validation cohort. This approach displays the advantage to provide external validity, to avoid overparameterization and to reduce the risk of type-1 error.

According to their distribution, continuous variables were provided as median with interquartile range (IQR) or as mean with standard deviations $( \pm S D)$. Student's t-test was used for their comparison. Categorical data were displayed as frequencies with valid percentages and compared with $\chi^{2}$ test. Receiver operating characteristic (ROC) curves were generated to calculate the area under the curve $(A \cup C)$. The threshold was determined to obtain equal sensitivity and specificity. Multivariable analyses integrating potential confounding factors were performed by logistic regression. 
TABLE 1 Cohorts and patients characteristics

\begin{tabular}{|llll|}
\hline & $\begin{array}{l}\text { Training } \\
\text { cohort } \\
(n=696)\end{array}$ & $\begin{array}{l}\text { Validation } \\
\text { cohort }(n=350)\end{array}$ & $p$ value \\
\hline Age (years) & $63(57-69)$ & $64(58-70)$ & .483 \\
\hline Gender (female) & $164(24)$ & $77(22)$ & .587 \\
\hline ASA score (III-IV) & $130(19)$ & $73(21)$ & .400 \\
\hline Smoking & $321(47)$ & $173(51)$ & .298 \\
\hline BMI (Kg/m $\left.{ }^{2}\right)$ & $26(22-29)$ & $26(23-29)$ & .675 \\
\hline Cancer type (ADC) & $424(61)$ & $216(62)$ & .966 \\
\hline Neoadjuvant therapy & & & .172 \\
\hline $\begin{array}{l}\text { Chemotherapy } \\
\text { Radiotherapy }\end{array}$ & $507(73)$ & $266(77)$ & .101 \\
\hline Surgery (Minimal invasive) & $347(50)$ & $193(55)$ & .857 \\
\hline $\begin{array}{l}\text { Laparoscopy } \\
\text { Thoracoscopy }\end{array}$ & $268(39)$ & $132(38)$ & .046 \\
\hline Surgery duration (min) & $94(14)$ & $63(19)$ & .660 \\
\hline Blood loss (ml) & $350(290-410)$ & $350(290-410)$ & $.600)$ \\
\hline
\end{tabular}

Note: Continuous variables are provided as median with interquartile range. Categorical variables are provided as frequencies with valid percentages.

Abbreviations: ADC, adenocarcinoma; ASA, American Society of Anesthesiologists; BMI, body mass index.

Variables with $p<.05$ in univariable analysis were further integrated and tested in multivariable analysis. Statistical significance was defined as a $p<.05$. IBM SPSS Statistics 25.0 was used to perform all analyses.

\section{3 | RESULTS}

\section{1 | Patients, cohorts, and outcomes}

A total of 1046 patients met the inclusion criteria and were attributed to either the training cohort $(n=696)$ or the validation cohort $(n=350)$. Patients' characteristics and the details of the surgery are provided in Table 1. These two cohorts were well balanced, displaying patients comparable for all variables, except for a higher rate of thoracoscopy in the validation cohort (19\% vs. $14 \%, p=.046$ ).

Altogether, minor, major, mortality, and overall complications were reported in 683 (65\%), $363(35 \%), 35$ (3\%), and 889 (85\%) patients, respectively. Median CCl was 20.9 (8.7-33.7) and median LoS was 15 days (11-24). The outcomes of each cohort are detailed in Table 2.

\subsection{Albumin profile and $\Delta$ Alb}

The perioperative profile of albumin is illustrated for the training (Figure 1A) and validation (Figure 1B) cohorts; albumin profiles were

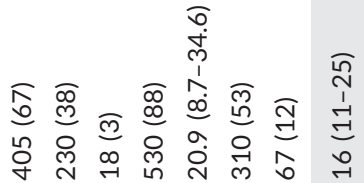

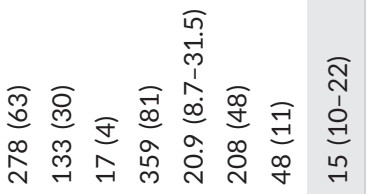

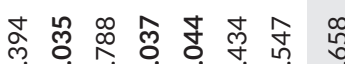

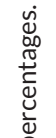

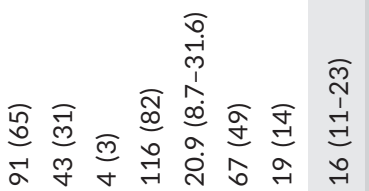

๙্ণ

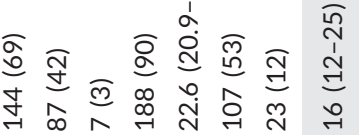
每 $\frac{\sqrt{3}}{5}$

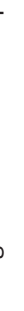


consistent in both cohorts. Esophagectomy triggered a prompt fall of albumin on POD 1, followed by a recovery phase. Patients who subsequently developed major complications showed a more rapid decrease in albumin.

Clustering of albumin levels per se on POD 1 did not statistically discriminate patients in terms of major complications rate (Figure 2).

The difference between preoperative and postoperative albumin values was measured on POD $1(\triangle \mathrm{Alb})$. Median $\triangle \mathrm{Alb}$ showed a consistent value of $11 \mathrm{~g} / \mathrm{L}(9-14)$ in all cohorts (training-, validation-, and whole-cohort). Patients with major complications showed higher $\Delta$ Alb in both training (12 vs. $11 \mathrm{~g} / \mathrm{L}, p=.022$ ) and validation (12 vs. $11 \mathrm{~g} / \mathrm{L}, p=.012$ ) cohorts (Figure 3 ).

\section{3 $\quad \Delta$ Alb cut-off and its capacity to discriminate adverse outcomes}

Using either technique to determine the ideal cut-off for major complications (either the median value or ROC curve for equal sensitivity/specificity), $\Delta$ Alb cut-off was consistently $11 \mathrm{~g} / \mathrm{L}$ in both training and validation cohorts. We next sought to test whether this cut-off was able to distinguish patients who will develop adverse outcomes (Table 2). $\Delta \mathrm{Alb} \geq 11 \mathrm{~g} / \mathrm{L}$ was associated with higher rates of overall complications in the training cohort ( 87 vs. $81 \%, p=.037$ ). In the validation cohort, patients with $\Delta \mathrm{Alb} \geq 11 \mathrm{~g} / \mathrm{L}$ showed increased major complications (42 vs. $31 \%$, $p=.035$ ), a higher rate of overall complications (90 vs. $82 \%$, $p=.037)$ as well as higher $\mathrm{CCl}(22.6$ vs. $20.9, p=.044)$. When merging the two cohorts, a difference was highlighted for major (38 vs. $30 \%, p=.007$ ) and overall complications (88 vs. $81 \%$, $p=.004)$. Sensitivity, specificity, positive predictive value (PPV), and negative predictive value (NPV) of $\triangle \mathrm{Alb}$ for major complications are summarized in Table $\mathrm{S} 1$.

(A)

Training cohort

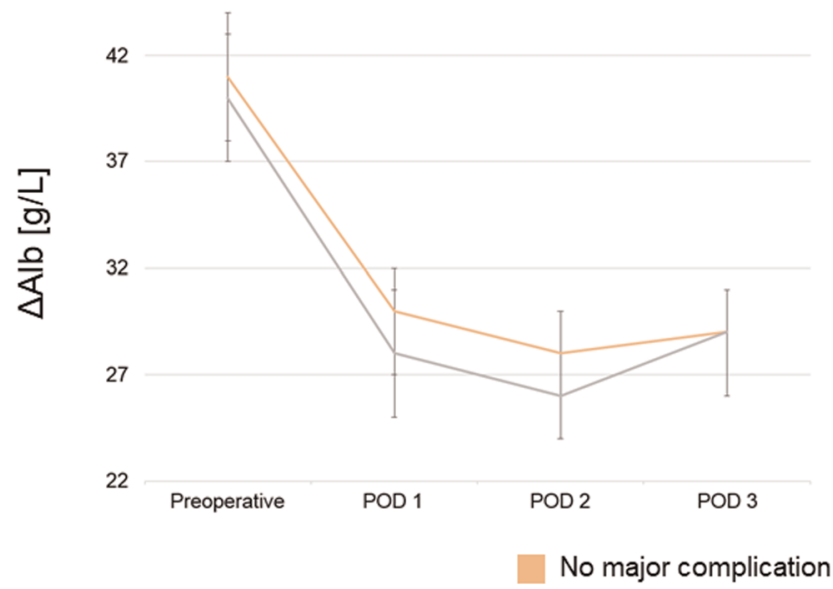

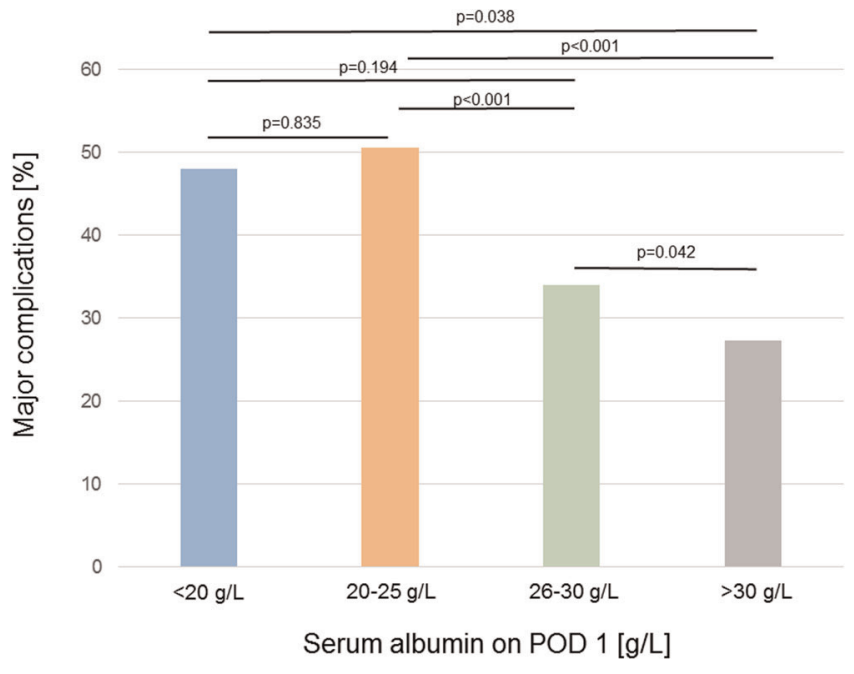

FIGURE 2 Incidence of major complications for clusters of albumin value on POD 1. Columns illustrate incidences of major complications ( $Y$-axis); patients were clustered based on their absolute value of serum albumin on POD 1 in [g/L] (X-axis): $<20 \mathrm{~g} / \mathrm{L}$ (blue), $20-25 \mathrm{~g} / \mathrm{L}$ (orange), $26-30 \mathrm{~g} / \mathrm{L}$ (green), and $>30 \mathrm{~g} / \mathrm{L}$ (gray). POD, postoperative day [Color figure can be viewed at wileyonlinelibrary.com]

\subsection{Predictors of major complications}

A logistic regression model integrating multiple potential confounding factors was performed to identify independent predictors of major complications (Table 3). In the training cohort, ASA score (OR, 1.78; 95\% $\mathrm{Cl}, 1.26-2.52 ; p=.001)$ and $\Delta \mathrm{Alb}(\mathrm{OR}, 1.06 ; 95 \% \mathrm{Cl}, 1.01-1.11 ; p=.014)$ were identified as independent predictors of major complications. In the validation cohort, $\triangle$ Alb was the only factor independently associated with major complications (OR, 1.05; 95\% Cl, 1.00-1.11; $p=.041)$. Of note, the absolute value of albumin POD 1 was not included in the multivariable analysis to avoid multicollinearity.

(B)

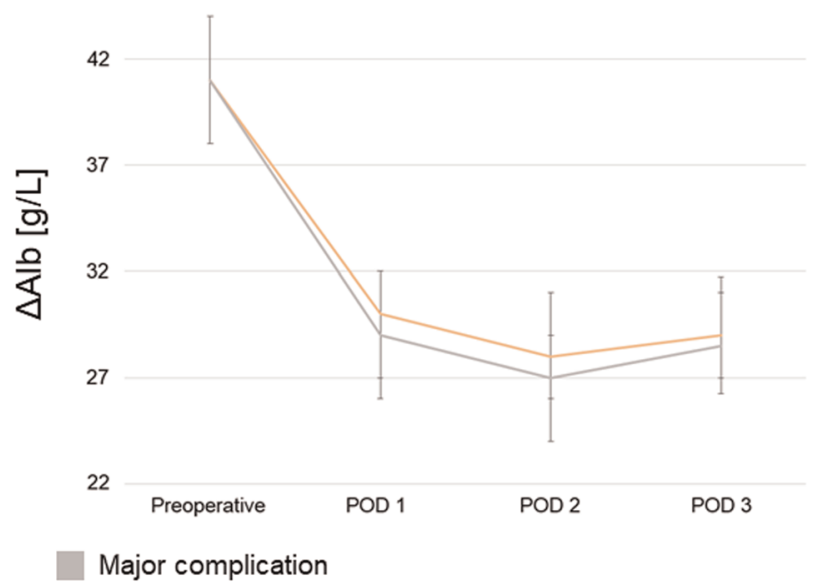

FIGURE 1 Perioperative profile of albumin. Perioperative profile of albumin: curves reflect median values of albumin [g/L] and bars represent interquartile range. Gray and orange curves indicate patients with and without major complications, respectively. A, Training cohort $(n=696)$. B, Validation cohort $(n=350)$ [Color figure can be viewed at wileyonlinelibrary.com] 


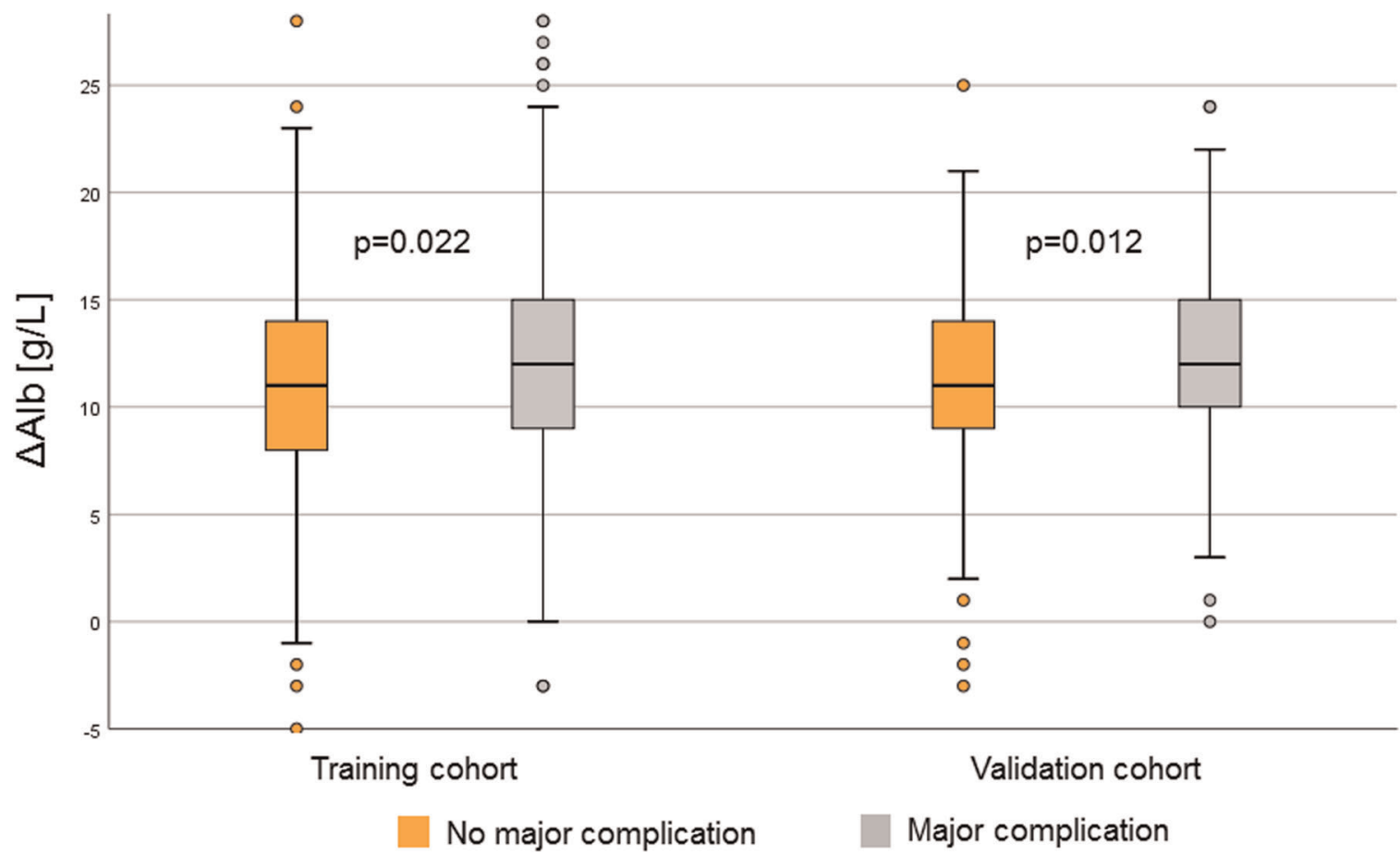

FIGURE 3 Comparison of $\triangle \mathrm{Alb}$. $\triangle \mathrm{Alb}$, boxplots of perioperative albumin variation ( $\triangle \mathrm{Alb})$. Gray and orange illustrate patients with and without major complications, respectively [Color figure can be viewed at wileyonlinelibrary.com]

TABLE 3 Multivariable analysis to identify independent predictors of major complications

\begin{tabular}{|c|c|c|c|c|c|c|c|c|c|c|c|c|}
\hline & \multicolumn{6}{|c|}{ Training cohort $(n=696)$} & \multicolumn{6}{|c|}{ Validation cohort $(n=350)$} \\
\hline & \multicolumn{3}{|c|}{ Univariable } & \multicolumn{3}{|c|}{ Multivariable } & \multicolumn{3}{|c|}{ Univariable } & \multicolumn{3}{|c|}{ Multivariable } \\
\hline & OR & $95 \% \mathrm{Cl}$ & $p$ value & OR & $95 \% \mathrm{Cl}$ & $p$ value & OR & $95 \% \mathrm{Cl}$ & $p$ value & OR & $95 \% \mathrm{Cl}$ & $p$ value \\
\hline Gender (female) & 0.59 & $0.41-0.85$ & .004 & 0.79 & $0.46-1.34$ & .383 & 0.98 & $0.58-1.65$ & 0.932 & & & \\
\hline ASA score & 1.29 & $1.03-1.63$ & .03 & 1.78 & $1.26-2.52$ & .001 & 1.09 & $0.79-1.50$ & 0.588 & & & \\
\hline BMI, $\mathrm{kg} / \mathrm{m}^{2}$ & 0.98 & $0.95-1.01$ & .192 & & & & 0.99 & $0.94-1.04$ & 0.709 & & & \\
\hline Cancer type (ADC) & 0.85 & $0.64-1.13$ & .257 & & & & 0.871 & $0.59-1.28$ & 0.483 & & & \\
\hline \multicolumn{13}{|l|}{ Neoadjuvant therapy } \\
\hline Chemotherapy & 0.83 & $0.58-1.17$ & .28 & & & & 0.65 & $0.39-1.07$ & 0.092 & & & \\
\hline Thoracoscopy & 1.19 & $0.76-1.88$ & .445 & & & & 0.57 & $0.31-1.04$ & 0.065 & & & \\
\hline Surgery duration, min & 1.00 & $1.00-1.01$ & .019 & 1.00 & $1.00-1.00$ & .159 & 1.00 & $1.00-1.01$ & 0.249 & & & \\
\hline Blood loss, ml & 1.00 & $1.00-1.00$ & .213 & & & & 1.00 & $1.00-1.00$ & 0.072 & & & \\
\hline Preoperative Alb, g/L & 0.98 & $0.95-1.00$ & .059 & & & & 1.00 & $0.96-1.05$ & 0.914 & & & \\
\hline$\Delta \mathrm{Alb}, \mathrm{g} / \mathrm{L}$ & 1.04 & $1.01-1.07$ & .023 & 1.06 & $1.01-1.11$ & .014 & 1.06 & $1.01-1.12$ & 0.013 & 1.05 & $1.00-1.11$ & 0.041 \\
\hline
\end{tabular}

Abbreviations: ADC, adenocarcinoma; ASA, American Society of Anesthesiologists; BMI, body mass index; $\mathrm{Cl}$, confidential interval; HR, hazard ratio. 
TABLE 4 Multivariable analysis to identify independent predictors of $\Delta \mathrm{Alb} \geq 11 \mathrm{~g} / \mathrm{L}$

\begin{tabular}{|c|c|c|c|c|c|c|c|c|c|c|c|c|}
\hline & \multicolumn{6}{|c|}{ Training cohort $(n=696)$} & \multicolumn{6}{|c|}{ Validation cohort $(n=350)$} \\
\hline & \multicolumn{3}{|c|}{ Univariable } & \multicolumn{3}{|c|}{ Multivariable } & \multicolumn{3}{|c|}{ Univariable } & \multicolumn{3}{|c|}{ Multivariable } \\
\hline & OR & $95 \% \mathrm{Cl}$ & $\underline{p}$ value & OR & $95 \% \mathrm{Cl}$ & $p$ value & OR & $95 \% \mathrm{Cl}$ & $p$ value & OR & $95 \% \mathrm{Cl}$ & $p$ value \\
\hline Gender (female) & 0.61 & $0.42-0.88$ & .007 & 0.68 & $0.45-1.03$ & .067 & 0.56 & $0.33-0.97$ & .039 & 0.68 & $0.37-1.22$ & .196 \\
\hline ASA score & 0.89 & $0.72-0.11$ & .311 & & & & 1.03 & $0.75-1.41$ & .85 & & & \\
\hline $\mathrm{BMI}, \mathrm{kg} / \mathrm{m}^{2}$ & 0.94 & $0.91-0.97$ & $<.001$ & 0.95 & $0.92-0.98$ & .003 & 0.94 & $0.89-0.98$ & .008 & 0.95 & $0.90-1.00$ & .038 \\
\hline Cancer type (ADC) & 0.69 & $0.53-0.90$ & .007 & 0.85 & $0.63-1.15$ & .286 & 0.63 & $0.43-0.93$ & .018 & 0.76 & $0.50-1.16$ & .202 \\
\hline \multicolumn{13}{|l|}{ Neoadjuvant therapy } \\
\hline Chemotherapy & 0.93 & $0.66-1.31$ & .676 & & & & 1.37 & $0.78-2.42$ & .271 & & & \\
\hline Thoracoscopy & 0.59 & $0.38-0.91$ & .017 & 0.75 & $0.44-1.28$ & .289 & 0.59 & $0.34-1.02$ & .058 & & & \\
\hline
\end{tabular}

Abbreviations: ADC, adenocarcinoma; ASA, American Society of Anesthesiologists; BMI, body mass index; Cl, confidential interval; HR, hazard ratio.

\subsection{Determinants of high $\Delta$ Alb}

A multivariable analysis aimed to explore and understand which factors were associated with an $\Delta \mathrm{Alb} \geq 11 \mathrm{~g} / \mathrm{L}$. Results were consistent in both cohorts, identifying higher BMI and laparoscopy as protective factors (Table 4).

\subsection{The predictive value of other inflammatory biomarkers for major morbidity}

A wider panel of commonly used inflammatory biomarkers (CRP, WBC) was available for a subset of 519 patients. Gender (OR, 0.49; $95 \% \mathrm{Cl}$, $0.27-0.87 ; p=.015)$, ASA (OR, 1.44; 95\% Cl, 1.02-2.03; $p=.039$ ), smoking status (OR, $0.47 ; 95 \% \mathrm{Cl}, 0.28-0.78 ; p=.003$ ), CRP on POD 3 (OR, 1.01; 95\% Cl, 1.00-1.01; $p<.001)$, and $\Delta \mathrm{Alb}(\mathrm{OR}, 1.06 ; 95 \% \mathrm{Cl}$, $1.00-1.11 ; p=.035$ ) were significantly associated with major morbidity in the training cohort. However, in the validation cohort, only $\Delta \mathrm{Alb}$ remained independently associated with major postoperative complications (OR, 1.08; 95\% Cl, 1.00-1.16; $p=.040$ ), outperforming all other variables including CRP and WBC values on POD 3 (Table S2).

\section{4 | DISCUSSION}

This study provided thorough and comprehensive data highlighting the role of albumin, particularly its early postoperative decrease $(\triangle \mathrm{Alb})$, to predict adverse postoperative outcomes in patients undergoing oncological esophageal resection.
As hypothesized, esophagectomy induced a rapid drop of albumin, 9,10,12,12,17 which appeared more pronounced in patients who subsequently developed major complications (Figure 1). Present analyses established a cut-off of $\Delta$ Alb at $11 \mathrm{~g} / \mathrm{L}$. This cut-off appeared to be robust, regardless of the methodology (dichotomization according to median value or with ROC curve for equal sensitivity/specificity) and of the cohort. Interestingly, the cut-off and predictive values seem to vary according to the type of surgery. In a prospective study on major abdominal surgery, our group identified an $\Delta \mathrm{Alb}$ of $10 \mathrm{~g} / \mathrm{L}$, calculated on POD 1 and predicting overall complications. ${ }^{12}$ One of the most important results from this study comes from the multivariable analysis, which integrated multiple potential confounding factors, where $\Delta \mathrm{Alb}$ was identified as an independent predictor of major complications in both cohorts, unlike other variables. This is likely explained by the fact that $\Delta$ Alb recapitulates the physiological stress intensity triggered by surgery, which is determined by several factors, such as the invasiveness of surgery and its duration, as well as the intrinsic characteristics of the patient. Therefore, $\Delta$ Alb outperforms these variables separately taken, when looking at complication prediction.

What factors determine the postoperative drop of albumin remains unclear. Laparoscopy and higher BMI appeared as protective factors, associated with lower decrease in albumin levels. Previous studies have also shown a reduction of albumin decrease on POD 1 after MIE compared to open resection, suggesting a blunted metabolic stress response with laparoscopy. ${ }^{22}$ The recently published MIRO study provides some further indirect evidence supporting this thesis, as a hybrid approach with a laparoscopic approach to the abdomen, and an open thoracotomy resulted in significantly lower rates of postoperative complications compared with open surgery. ${ }^{6}$ 
Previous studies support that capillary leak of albumin from plasma to adjacent tissues is the main source of albuminemia decrease, along with reduced hepatic production shifting to acute-phase proteins, such as CRP. ${ }^{17}$ Why a higher BMI impacts on albumin levels is unclear. A higher BMI was not associated with higher baseline levels and whether a high BMI, possibly in association with nonalcoholic fatty liver disease, blunts the postoperative switch between the acute phase response and generation of constitutive proteins, such as albumin, requires further study. BMI alone moreover does not fully reflect the nutritional status, and other surrogate parameters such as sarcopenia would be helpful, but were not available in the present study. Nonetheless, the present study was not designed to investigate the complex physiological mechanisms of albumin fall and thus we cannot speculate on this point.

In the only comparable study, Ryan et al. in 2007 investigated the postoperative hypoalbuminemia in patients undergoing esophagectomy, and used categorical levels on Day 1 rather than $\Delta \mathrm{Alb}$, and severe hypoalbuminemia was associated with increased risk of major complications. ${ }^{14}$ However, $\Delta$ Alb may be more informative since it is a dynamic value calculated as a difference between preoperative and postoperative values of albumin. $\Delta \mathrm{Alb}$ as a biomarker of complication risk has an appealing rationale. These include its early indicative value, on POD 1, its ease to measure, reproducibility, and inexpensive cost. To date, $\Delta$ Alb has been reported to be associated with overall complications. ${ }^{10,12}$ This study highlighted its value for anticipating major adverse events, an important stake of postoperative course in major surgery. ${ }^{18}$

Several other biomarkers have been assessed for their capacity to predict postoperative complications; however, none of them has been widely implemented in daily clinical practice, as specific thresholds have yet to be validated. In addition, most of these biomarkers suffer from slow kinetics and are informative at a later time postoperatively (POD 2-4) compared with $\triangle$ Alb on Day 1 . Serum CRP has been identified as a negative predictor of anastomotic leakage with the best diagnostic accuracy at POD 2, 3, and $6 .{ }^{23}$ Published cut-offs for CRP show a wide range from $>200 \mathrm{mg} / \mathrm{ml}$ on $\mathrm{POD} 2^{18}$ or $>209 \mathrm{mg} / \mathrm{ml}$ on POD $2^{23}$ to $>111 \mathrm{mg} / \mathrm{L}$ on POD $4 .{ }^{24}$ Thus, while CRP levels are commonly measured in daily practice, there is still no universally accepted threshold to detect postoperative complications. In the present study, CRP levels on POD 3 were predictive of major morbidity in the training, but not the validation cohort (Table S2). Although it was only available for a subset of patients, and more large-scale data might be helpful, its predictive value was less than $\Delta \mathrm{Alb}$ in multivariable analysis. Previous studies have also identified hyperbilirubinemia as a risk factor for postoperative infectious complications, ${ }^{24,25}$ while serum angiotensin-converting enzyme 2 levels from POD 0-3 predicted postoperative pneumonia. ${ }^{26}$ However, these biomarkers are not commonly used in current practice after esophageal surgery and were, thus, not analyzed in this cohort.

Limitations of this study are mainly related to its retrospective design. Parameters such as perioperative fluid management, a potential confounder of serum albumin concentration, is not available for this series. Inclusion of a relatively recent series of patients in reference centers aimed to limit heterogeneity of perioperative care protocols; however, precise and reliable information of fluid perfusion is unfortunately difficult to obtain retrospectively. Moreover, (I) involved centers use clinical pathways that include goaldirect fluid therapies and (II) data have demonstrated that fluid minimally contributed to albumin decrease. ${ }^{12,15,17}$ The strengths of this study, however, include a multicenter design, including five highvolume centers, large sample size, and analysis of training and validation cohorts. Importantly, the design of the present study-with both a training and a validation cohort-precludes from a double (in the training cohort and thereafter in the validation cohort) type-1 error. In other words, these findings on $\Delta \mathrm{Alb}$ are highly unlikely fortuitous. Another potential drawback might be the timing of postoperative albumin values. In this study, it was not available on POD 0 (4-6 h after the end of surgery) after esophageal resection. In liver surgery, where albumin is often measured on that timepoint, $\triangle$ Alb could be calculated on POD 0 and was more performant than on POD $1 .^{13}$ Measuring albumin on POD 0 could be an option to optimize $\triangle \mathrm{Alb}$ and enhance its performance, in esophageal surgery, as previous data from our institution have suggested. ${ }^{17}$

These findings provide a solid rationale to further investigate $\Delta$ Alb in esophageal surgery and understand how this marker may guide decision-making in the early and critical postoperative phase. Early identification of patients at increased risk allows tailored individual surveillance with early imaging or endoscopy, whereas $\Delta \mathrm{Alb}$ may permit to select patients who would benefit from specific pharmaceutical measures, although such interventions have yet to be explored by randomized clinical trials. Of note, one can easily challenge a novel biomarker, such as $\Delta \mathrm{Alb}$, questioning its clinical contribution. The impact of $\Delta \mathrm{Alb}$ in clinical practice is at least as important as any other markers, such as CRP, which are extensively used worldwide in daily routine, except that $\Delta$ Alb is informative even earlier.

In summary, $\Delta \mathrm{Alb}>11 \mathrm{~g} / \mathrm{L}$ on POD 1 appeared as a valuable biomarker to predict major complications after esophagectomy. Further prospective trials are needed to define how precisely it may be integrated to guide postoperative management of patients undergoing surgery.

\section{CONFLICTS OF INTERESTS}

Mark I. van Berge Henegouwen: Researcher initiated grants Olympus and Stryker, consultant Mylan Medtronic and Johnson and Johnson. All fees paid tolnstitution and not related to present study. Guillaume Piessen: Relevant Financial Activities Outside the Submitted Work - AMGEN, ROCHE.

\section{AUTHOR CONTRIBUTIONS}

Study concept and design: Ismail Labgaa, Styliani Mantziari, Pierre Allemann, Martin Hübner, Nicolas Demartines, Markus Schäfer. Acquisition of data: Maxime Genety, Jessie A. Elliott, Satoshi Kamiya, Marianne C. Kalff, Michaël Winiker, Marguerite Messier. Analysis and interpretation of data: Ismail Labgaa, Styliani Mantziari, Jérôme Pasquier, Mark I. van Berge Henegouwen, Magnus Nilsson, John V. Reynolds, Guillaume Piessen, Markus Schäfer. Drafting of the manuscript: Ismail Labgaa, Styliani Mantziari, Markus Schäfer. Critical revision of the manuscript for important intellectual content: Ismail Labgaa, Styliani 
Mantziari, Maxime Genety, Jessie A. Elliott, Satoshi Kamiya, Marianne C. Kalff, Michaël Winiker, JP, Pierre Allemann, Marguerite Messier, Mark I. van Berge Henegouwen, Magnus Nilsson, John V. Reynolds, Guillaume Piessen, Martin Hübner, Nicolas Demartines, Markus Schäfer.

\section{SYNOPSIS}

This multicenter study investigated the early postoperative decrease of serum albumin $(\triangle \mathrm{Alb})$ as predictor of adverse outcomes after esophageal surgery. $\Delta$ Alb appeared as an independent predictor of major complications and as a promising biomarker in patients undergoing esophagectomy for cancer.

\section{DATA AVAILABILITY STATEMENT}

Data are available on request from the authors.

\section{ORCID}

Ismail Labgaa (D) https://orcid.org/0000-0003-4286-2170

Styliani Mantziari (D) https://orcid.org/0000-0003-1315-1898

Nicolas Demartines (D) http://orcid.org/0000-0002-1530-3114

\section{REFERENCES}

1. Pennathur A, Gibson MK, Jobe BA, Luketich JD. Oesophageal carcinoma. Lancet. 2013;381(9864):400-412.

2. Fitzmaurice $C$, Dicker $D$, Pain $A$, et al. The global burden of cancer 2013. JAMA Oncol. 2015;1(4):505-527.

3. Lagergren J, Smyth E, Cunningham D, Lagergren P. Oesophageal cancer. Lancet. 2017;390(10110):2383-2396.

4. Lordick F, Mariette C, Haustermans K, et al. Oesophageal cancer: ESMO clinical practice guidelines for diagnosis, treatment and follow-up. Ann Oncol. 2016;27(suppl 5):v50-v57.

5. Biere SS, van Berge Henegouwen MI, Maas KW, et al. Minimally invasive versus open oesophagectomy for patients with oesophageal cancer: a multicentre, open-label, randomised controlled trial. Lancet. 2012;379(9829):1887-1892.

6. Mariette C, Markar SR, Dabakuyo-Yonli TS, et al. Hybrid minimally invasive esophagectomy for esophageal cancer. N Engl J Med. 2019; 380(2):152-162.

7. Fransen LFC, Berkelmans GHK, Asti E, et al. The effect of postoperative complications after minimally invasive esophagectomy on long-term survival: an International Multicenter Cohort Study [published online ahead of print January 14, 2020]. Ann Surg.

8. Markar S, Gronnier C, Duhamel A, et al. The impact of severe anastomotic leak on long-term survival and cancer recurrence after surgical resection for esophageal malignancy. Ann Surg. 2015;262(6):972-980.

9. Liou DZ, Serna-Gallegos D, Mirocha J, Bairamian V, Alban RF, Soukiasian HJ. Predictors of failure to rescue after esophagectomy. Ann Thorac Surg. 2018;105(3):871-878.

10. Labgaa I, Demartines N, Hubner M. Biomarkers capable to early predict postoperative complications: the grail. Ann Surg. 2017; 266(6):e91-e92.

11. Labgaa I, Demartines N, Hubner M. Serum albumin: a promising biomarker to anticipate postoperative complications. Dis Colon Rectum. 2017;60(9):e616.

12. Hübner M, Mantziari S, Demartines N, Pralong F, Coti-Bertrand P, Schäfer M. Postoperative albumin drop is a marker for surgical stress and a predictor for clinical outcome: a pilot study. Gastroenterol Res Pract. 2016;2016:8743187-8743188.

13. Labgaa I, Joliat GR, Demartines N, Hübner M. Serum albumin is an early predictor of complications after liver surgery. Dig Liver Dis. 2016;48(5):559-561.

14. Ryan AM, Hearty A, Prichard RS, Cunningham A, Rowley SP, Reynolds JV. Association of hypoalbuminemia on the first postoperative day and complications following esophagectomy. J Gastrointest Surg. 2007;11(10):1355-1360.

15. Labgaa I, Joliat GR, Kefleyesus A, et al. Is postoperative decrease of serum albumin an early predictor of complications after major abdominal surgery? A prospective cohort study in a European centre. BMJ Open. 2017;7(4):e013966.

16. Liu ZJ, Ge XL, Ai SC, et al. Postoperative decrease of serum albumin predicts short-term complications in patients undergoing gastric cancer resection. World J Gastroenterol. 2017;23(27):4978-4985.

17. Mantziari S, Hübner M, Coti-Bertrand P, Pralong F, Demartines N, Schäfer M. A novel approach to major surgery: tracking its pathophysiologic footprints. World J Surg. 2015;39(11):2641-2651.

18. Babic B, Tagkalos E, Gockel I, et al. C-reactive protein levels after esophagectomy are associated with increased surgical trauma and complications. Ann Thorac Surg. 2020;109:1574-1583.

19. Dindo D, Demartines N, Clavien PA. Classification of surgical complications: a new proposal with evaluation in a cohort of 6336 patients and results of a survey. Ann Surg. 2004;240(2):205-213.

20. Slankamenac K, Graf R, Barkun J, Puhan MA, Clavien PA. The comprehensive complication index: a novel continuous scale to measure surgical morbidity. Ann Surg. 2013;258(1):1-7.

21. Hastie T, Tibshirani R, Friedman J. The Elements of Statistical Learning. Springer Series in Statistics. New York: Springer; 2001:222.

22. Scarpa M, Cavallin F, Saadeh LM, et al. Hybrid minimally invasive esophagectomy for cancer: impact on postoperative inflammatory and nutritional status. Dis Esophagus. 2016;29(8):1064-1070.

23. Gordon AC, Cross AJ, Foo EW, Roberts RH. C-reactive protein is a useful negative predictor of anastomotic leak in oesophago-gastric resection. ANZ J Surg. 2018;88(3):223-227.

24. Miki $\mathrm{Y}$, Toyokawa $\mathrm{T}$, Kubo $\mathrm{N}$, et al. C-reactive protein indicates early stage of postoperative infectious complications in patients following minimally invasive esophagectomy. World J Surg. 2017;41(3):796-803.

25. Muneoka $\mathrm{Y}$, Ichikawa $\mathrm{H}$, Kosugi $\mathrm{S}$, et al. Hyperbilirubinemia predicts the infectious complications after esophagectomy for esophageal cancer. Ann Med Surg. 2019;39:16-21.

26. Nomura S, Tsujimoto $\mathrm{H}$, Aosasa $\mathrm{S}$, et al. Impact of angiotensinconverting enzyme 2 levels on postoperative pneumonia after esophagectomy. J Surg Res. 2018;224:200-206.

\section{SUPPORTING INFORMATION}

Additional Supporting Information may be found online in the supporting information tab for this article.

How to cite this article: Labgaa I, Mantziari S, Genety M, et al. Early postoperative decrease of albumin is an independent predictor of major complications after oncological esophagectomy: A multicenter study. J Surg Oncol. 2021;123:462-469. https://doi.org/10.1002/jso.26317 